\title{
Optimal management of endemic Burkitt lymphoma: a holistic approach mindful of limited resources
}

This article was published in the following Dove Press journal:

Blood and Lymphatic Cancer: Targets and Therapy

23 September 2014

Number of times this article has been viewed

\author{
Ann M Moormann',* \\ Jodi L Skiles ${ }^{2}$,* \\ Juliana A Otieno ${ }^{3}$ \\ Geoffrey C Buckle' \\ Terry A Vik ${ }^{2}$
}

'University of Massachusetts Medical School, Worcester, MA, USA; ${ }^{2}$ Indiana University School of Medicine, Indianapolis, IN, USA; 3 Jaramogi Oginga Odinga Teaching and Referral Hospital, Ministry of Medical Services, Kisumu, Kenya

*These authors contributed equally to this work
Correspondence: Ann M Moormann University of Massachusetts Medical School, 373 Plantation Street, Biotech 2, Suite 318, Worcester, MA 01605, USA $\mathrm{Tel}+\mathrm{I} 5088568826$

Fax + I 5088565500

Email ann.moormann@umassmed.edu
Abstract: Endemic Burkitt lymphoma (BL) was publically described in 1958 and remains the most prevalent pediatric cancer in equatorial Africa with an annual incidence between two and five cases per 100,000 children. Several risk factors have been identified, including early-age infection with Epstein-Barr virus, a geographic association with high Plasmodium falciparum malaria transmission, and poor nutrition. However, other modifiable factors play a role in the survival of these children. Treatment regimens for BL have evolved over a time period that spans nearly 50 years. This review will compare survival between different combination chemotherapeutic regimens and discuss other key determinants of outcomes among children diagnosed with BL in resource-limited settings. A discussion of obstacles to diagnosis will be presented, including low community awareness of pediatric cancer, limited access to health facilities, inaccurate or delayed diagnosis often beginning at lower level health rural facilities that are typically staffed by those with limited training in oncology, and insufficient pathology support to confirm diagnosis. Other challenges examined here include those related to treatment adherence, specifically the social and economic stressors that can lead to abandonment of care, and treatment-related toxicity, a challenge compounded not only by the scarcity of medications for supportive care, but also the paucity of clinically trained medical professionals available to manage integrated care for these children. Future directions for enhancing BL survival will be discussed including molecular approaches for rational drug discovery as well as the benefits of forming a unified, global BL research network.

Keywords: pediatric cancer, treatment regimen, Africa, social and economic predictors, biomarkers

\section{Introduction}

Burkitt lymphoma (BL) is a mature B-cell neoplasm of the lymphatic system that was publically described in 1958 by a surgeon named Denis Burkitt who was working in equatorial Africa at the time. ${ }^{1}$ He documented a clinical entity that characteristically affects the jaw or other facial bones of young children. ${ }^{1}$ It is now known that BL also affects other sites, including the abdomen (lymph nodes, spleen, kidneys, liver, ovaries), extradural space of the spinal canal (often presenting as paraplegia), bone marrow, and central nervous system. One of the key clinical characteristics of $\mathrm{BL}$ is that it rapidly progresses and can double in size within 48 hours, and therefore requires urgent evaluation and treatment. ${ }^{2}$ However, the rapid doubling time of these tumor cells make them exquisitely responsive to chemotherapy without the need for radiotherapy or surgery. This also makes this cancer imminently curable in resourcelimited settings where it is most prevalent. 
$\mathrm{BL}$ is widely reported to be the most common pediatric cancer in Africa. ${ }^{3,4}$ The average annual incidence of endemic $\mathrm{BL}$ (eBL) is two cases per 100,000 children, which is 20 -fold higher than sporadic BL in the US. ${ }^{3,5}$ The median onset age of eBL is 6 years (ranging from $2-14$ years of age) ${ }^{6}$ and coinfections with Epstein-Barr virus (EBV) and Plasmodium falciparum malaria early in life have been identified as risk factors in the development of this pediatric cancer (reviewed in Moormann et al). ${ }^{7}$

Despite over 50 years of intensive clinical study and epidemiologic research, BL remains the most prevalent pediatric cancer in equatorial Africa and survival rates for children receiving treatment remain far below those in high-income countries. Improving outcomes in this setting requires a greater understanding of both how to deliver the chemotherapeutic armamentarium at hand and ways to apply evidence based medical practice for evaluating new chemotherapies. Additionally, there is a need to more broadly and effectively address many of the unique challenges of managing cancer therapy in resource-limited settings, which includes not just the medical aspects of diagnosis and treatment, but also the social and economic considerations that can lead to poor outcomes, largely through abandonment of care. This review goes beyond the bedside to describe other factors critical to achieving the optimal management of BL and how they could be implemented.

\section{Epidemiology and etiology}

BL was the first human cancer demonstrated to harbor a virus. ${ }^{8}$ This discovery by Anthony Epstein, Yvonne Barr, and Bert Achong led to implicating EBV and other viruses in the etiology of human cancers. Since its discovery, the understanding of EBV persistence, pathogenesis, and immune evasion that facilitate the development of malignant B-cells has made great strides even if the precise mechanisms have yet to be fully realized. The roles of EBV gene products and the $c-m y c$ translocation in $\mathrm{BL}$ pathogenesis have been reviewed in detail elsewhere. ${ }^{9}$

BL was also the first human cancer geographically mapped by its incidence. In 1961, Denis Burkitt, Ted Williams, and Cliff Nelson were able to localize BL incidence in Africa to the lymphoma belt that straddles the equator by 15 degrees to the north and south. ${ }^{10,11}$ This observation led Alexander Haddow to speculate that a mosquito vector might be involved in eBL etiology, ${ }^{12}$ which prompted Gilbert Dalldorf in 1964 to hone in on intense $P$. falciparum malaria transmission as a risk factor for eBL. ${ }^{13}$ The geographic overlay between high malaria transmission and incidence of eBL has been previously described ${ }^{14,15}$ and validated by others, ${ }^{16,17}$ however, the precise mechanisms by which malaria contributes to eBL pathogenesis remain speculative and are reviewed in more detail by Moormann et al. ${ }^{7}$

In brief, early studies suggested that malaria impaired T-cell control over EBV-infected B-cells ${ }^{18,19}$ and also induced lytic reactivation. ${ }^{20}$ Over the past 40 years, it has become possible to appreciate the complexity of human immune responses to infectious diseases and the necessity of longitudinal infant cohort studies to demystify the sequence and magnitude of events that result in inefficient immunity to EBV. 7 The working hypothesis for eBL etiology is two-fold and synergistic. In populations where eBL is prevalent, nearly all children experience their primary EBV infection before 1 year of age. ${ }^{21}$ This suggests that maternal antibodies are not effectively protecting infants from early-age EBV infections. This time period also spans the age at which children are most susceptible to malaria if maternal antibodies are insufficiently protective. In malaria holoendemic areas, children can experience two to three malaria infections during the first year of life, but after cumulative infections children develop partially protective immunity that allows them to harbor asymptomatic infections..$^{22}$ This immunologic tolerance allows the sustained interaction between malaria and EBV within the human host with evidence of T-cell exhaustion (Moormann, unpublished data, 2013) after this prolonged attempt to balance immune control over viral pathogenesis.

Understanding precisely how these two ubiquitous childhood infections lead to oncogenesis is foreseeable, provided investments are made in robust epidemiologic studies that are designed with the appropriate control group comparisons to answer these questions. Conclusions about the role of malaria in eBL etiology can only be made when controls are matched to cases by variables that impact malaria exposure and resulting immunity. These variables include geography and chronology, which control for malaria transmission intensity and parasite genetic diversity, and ethnicity and age, which may influence immunity to both malaria and EBV. Challenges would nevertheless remain as to how to prevent these common childhood infections in the endemic setting and thereby prevent eBL. In addition, the possibility remains that even with malaria control, BL could remain incident. Other cofactors may replace malaria as the immune modulating event given EBV is the initiator of tumorigenesis. Prior studies have explored the role of nutritional deficiencies, ${ }^{23}$ arboviral infections as other risk factors, ${ }^{24}$ and human immunodeficiency virus (HIV), particularly in the setting of a growing generation of HIV-infected infants ${ }^{25}$ who are surviving longer on 
antiretroviral therapy. Immunodeficiency-associated BL has been documented for adult HIV patients, ${ }^{26}$ and children who are HIV infected and present with BL have a worse prognosis compared to those who do not have HIV. ${ }^{27,28}$ There is also the possibility that age of primary EBV infection might naturally shift to an older age group with improving socioeconomic conditions in developing countries. The changing epidemiology of EBV has been recently demonstrated in populations within the US $\mathrm{US}^{29}, 30$ and discussions are currently underway on the feasibility of testing a prophylactic EBV vaccine. ${ }^{31}$ The authors speculate that the global roll out of malaria control programs and improvements in infrastructure may explain the gradual decrease in eBL incidence observed at the Jaramogi Oginga Odinga Teaching and Referral Hospital in Kisumu, Kenya over the past 7 years. Western Kenya is the catchment area for this regional cancer referral hospital, and also the area where the Kenya Medical Research Institute, in collaboration with the US Centers for Disease Control and Prevention, conducted the original insecticide-treated bed net trials that demonstrated sustained reductions in malaria transmission. ${ }^{32}$ Unfortunately, malaria may be resurging due to changes in vector behaviors ${ }^{33}$ and mosquito resistance to permethrin that is used to impregnate the bed nets. ${ }^{34}$

\section{Diagnosis}

The challenges in diagnosing cancers in low-income settings are many and multifactorial. Community awareness of cancer in these settings is often limited. ${ }^{35}$ Many patients who have characteristic findings of BL (eg, jaw mass, distended abdomen, fevers, weight loss, night sweats) will initially be evaluated by an herbalist, traditional healer, or by practitioners in a rural clinic setting who may not consider the possibility of a cancer diagnosis. ${ }^{36}$ Consequently, precious time can be wasted with various treatments that are ultimately futile, resulting in delays in necessary medical consultation, diagnostic evaluation, and initiation of appropriate treatment. Furthermore, as in most settings, access to health services remains limited among the poorest populations. Even when a cancer diagnosis is recognized and referred immediately, economic barriers and lack of transportation to an equipped tertiary facility may delay further evaluation by weeks or even months.

Once a child has been appropriately referred and evaluated at a tertiary care level facility, there are still several barriers that must be overcome to make a definitive diagnosis of BL. Although the clinical presentation of BL has characteristic clues that may lead to a high clinical suspicion of BL, making a tissue-based histopathological diagnosis is critical to both ensure accurate diagnosis (to exclude other primary tumors) and inform decisions regarding appropriate treatment. In settings with plentiful resources, the diagnosis of BL is confirmed with a multimodal approach of histopathology, including immunohistochemistry on a tissue specimen obtained by biopsy (incision, excisional, or core), flow cytometry, and cytogenetics. Unfortunately, this combination of technology and resources rarely exists in resource-limited settings. In the unlikely scenario that they do exist, the turnaround time for diagnosis (estimates of 7-10 days) palpably lags in the race to treat rapidly growing tumors such as BL. Not only do resource-limited settings often lack the technology to carry out necessary diagnostics, but the lack of personnel trained in these modalities and logistical limitations, such as access to a surgical theater or appropriate anesthesia to obtain a biopsy specimen, abound as well. ${ }^{35}$

Consequently, the diagnosis of BL is often based solely on the child's clinical presentation and may or may not include fine-needle aspiration biopsy with cytologic examination. Fine-needle aspiration biopsy is a diagnostic modality that offers relatively rapid results, often available within 24-48 hours; however, its accuracy is not optimal. A recent multi-institutional assessment of fine-needle aspiration biopsy for childhood cancers in resource-limited settings demonstrated a $96.4 \%$ diagnostic accuracy for nonHodgkin's lymphoma, with $87.5 \%$ (14/16 cases) accurately diagnosed as BL. ${ }^{37}$ Unfortunately, this approach may lead to misdiagnosis in some children, which can have devastating implications, all too often leading to poor outcomes in a disease that would otherwise be curable. Some have advocated for an Internet-based telemedicine approach for pathologic consultation to address the need for trained pathologists; however, lack of reliable internet resources and costs associated with implementing this technology have prohibited widespread adoption. Optimism is being renewed with decreasing costs of mobile devices such as open-source tablets and cell phones, which are now being used to store and send digital images for remote consultations and for educational training purposes with other forms of cancer. ${ }^{38} \mathrm{In}$ addition, concerted efforts to develop and strengthen national cancer registries in sub-Saharan countries have arisen, particularly pertaining to HIV and acquired immunodeficiency syndrome-related cancers. ${ }^{39}$

Following a proper diagnosis of BL, every child should also undergo staging of the tumor in order to accurately assess the extent of disease. In resource-rich settings, disease extent informs treatment decisions such that more advanced tumors are treated more aggressively and less advanced tumors require 
less intensive treatment. Ideally, disease staging should include chest X-ray to assess for mediastinal involvement and/or the presence of pleural effusion, abdominal ultrasound to evaluate for intraabdominal lymphadenopathy and/or organomegaly, bone marrow aspiration and biopsy to evaluate for malignant infiltration of the bone marrow space, and a spinal tap with cerebrospinal fluid cytology to assess for evidence of central nervous system involvement. Again, due to lack of resources and trained personnel and logistical limitations, this staging evaluation is rarely undertaken or is often incomplete or imprecise in most resource-limited settings. Without full information on staging, many children with BL in resource-limited settings are treated with a standardized one-size-fits-all protocol without consideration of risk factors, including extent of disease. Thus, children may receive more or less aggressive therapy than their disease would otherwise mandate if they were being treated in a high-income setting. Alternatively, a lower induction dose of chemotherapy may be prescribed for children with larger palpable tumors in an attempt to mitigate tumor lysis syndrome. While these practices may initially appear substandard, this approach has its merits in settings where treatment options and supportive care measures are less sophisticated. Moreover, prognostic factors that have been validated in high-income countries may have reduced predictive value in low-income countries where most patients have advanced disease due to late presentation, and other comorbidities, including malnutrition and infections, are abundant. ${ }^{4}$ There is clearly a need to discover predictive and prognostic biomarkers for BL that could be readily measured from serum samples and used to guide treatment. Lactate dehydrogenase (LDH) has been used as a nonspecific surrogate of tumor burden; however, serum $\mathrm{LDH}^{40}$ may be suboptimal in settings where children experience malaria coinfections that are independently associated with elevated LDH levels. ${ }^{41}$ Discovering biomarkers with high sensitivity and specificity for eBL would enable a rational approach for identifying which children to put "on trial"42 and those who may do just fine using the conventional, less intensive treatment.

\section{History of treatment regimens and patient survival}

$\mathrm{BL}$ is a rare example of pediatric cancer that can be cured with a short course or even single dose of cyclophosphamide. Multiple studies reported in the 1960s and 1970s showed survival rates of $6 \%-38 \%$ for large numbers of African children with eBL. ${ }^{43-52}$ Based on those early findings and the concerns for increased toxicity with combination chemotherapy, treatment for BL in Africa has remained unchanged for many years. After reports of improved survival in limited-stage disease in children treated with combination chemotherapy, including cyclophosphamide, vincristine, methotrexate, and prednisone (COMP), on a North American Children's Cancer Group (CCG) trial, CCG-551, ${ }^{53}$ and marked improvement in outcomes for children with both limited- and advanced-stage BL treated with combination chemotherapy on the lymphoma malignancy B-cell (LMB) trials, ${ }^{54}$ new treatment protocols were introduced in Africa. Reports from ten trials published since 2000 are summarized in Table 1.

The underlying question "what is the optimal treatment for eBL?" is difficult to answer based on underlying differences between studies. Issues limiting a meta-analysis include patient selection bias, different inclusion and exclusion criteria, adequacy of diagnosis and staging, varying levels of treatment adherence, differences in outcomes reported, and adequacy of reporting of study methodology. What is apparent from Table 1 is that there is a trend toward improved survival with increasing intensity of treatment. Single agent trials achieved 30\%-50\% survival, while studies using COMP as the backbone of treatment reported survival rates of $50 \%-70 \%$. Centers able to administer treatment most similar to the LMB intermediate risk therapy with doxorubicin, higher dose methotrexate, and cytarabine had survival rates of $75 \%-80 \%$. The authors' experience in Eldoret, Kenya within the Academic Model for Providing Access to Healthcare (AMPATH) program over the past 4 years delivering therapy including doxorubicin and cytarabine on a backbone of COMP therapy mirrors that overall outcome. The authors' experience in Kisumu at the Jaramogi Oginga Odinga Teaching and Referral Hospital using COMP over the past 10 years had survival rates of $67 \%$. While more intensified treatments have led to improved outcomes, the role of robust ancillary care such as antibiotics, nutritional support, and blood products cannot be overemphasized. Previous efforts to introduce high-dose intensive treatment protocols with limited supportive care resources have resulted in unacceptably high treatment-related morbidity and mortality. ${ }^{55}$ Unfortunately, many centers have inadequate infrastructure and limited capacity to deliver this care. Both areas must be strengthened in order to administer intensive therapy for BL tumors that are advanced stage at diagnosis or refractory to conventional therapy. To attain survival rates similar to those in high-income countries, concerted effort will be needed to focus on improving not only the infrastructure, but also the knowledge base of medical practitioners throughout the health system, including lower level health 
Table I Dose intensity of chemotherapeutic agents used in ten protocols of treatment for Burkitt lymphoma in Africa reported since 2000

\begin{tabular}{|c|c|c|c|c|c|c|c|c|c|c|}
\hline \multicolumn{11}{|c|}{ Chemotherapy dose intensity ( $\mathrm{mg} / \mathrm{m}^{2} /$ week) } \\
\hline $\begin{array}{l}\text { Study } \\
\text { type }\end{array}$ & $\begin{array}{l}\text { Citation (first } \\
\text { author, year) }\end{array}$ & Cyclophosphamide & Prednisone & Vincristine & Methotrexate & Cytarabine & Doxorubicin & $\begin{array}{l}\text { IT } \\
\text { meds }\end{array}$ & Survival & Patients \\
\hline Single & Kazembe, ${ }^{65} 2003$ & 600 & & & & & & No & $43 \%$ & 92 \\
\hline \multirow[t]{2}{*}{ agent } & Traore, ${ }^{66} 2011$ & 1,200 & & & & & & Yes & $33 \%$ & 178 \\
\hline & Hesseling, ${ }^{67} 2009$ & $600-1,200$ & & & & & & Yes & $48 \%$ & 40 \\
\hline \multirow[t]{9}{*}{ COMP } & Koffi, ${ }^{68} 2010$ & $\mathrm{I}, 200$ & & & 30 & 250 & & Yes & $62 \%$ & 85 \\
\hline & Ngoma, ${ }^{69} 2012$ & 600 & & 0.7 & 37.5 & & & Yes & $62 \%$ & 356 \\
\hline & Hesseling, ${ }^{70} 2005$ & 250 & 210 & 1 & 1,000 & & & Yes & $33 \%$ & 42 \\
\hline & Hesseling, ${ }^{55} 2003$ & 250 & 210 & 1 & 1,000 & 200 & & Yes & $59 \%$ & 44 \\
\hline & Davidson, ${ }^{71} 2010$ & 300 & 75 & 0.75 & 75 & & & Yes & $72 \%$ & 29 \\
\hline & Hesseling, ${ }^{72} 2012$ & 1,200 & & 0.5 & 333 & & & Yes & $61 \%$ & 129 \\
\hline & Traore, ${ }^{66} 2011$ & 500 & 100 & 0.67 & 1,000 & & & Yes & $50 \%$ & 76 \\
\hline & Harif, $^{73} 2008$ & 500 & 100 & 0.67 & 1,000 & & & Yes & $56 \%$ & 187 \\
\hline & & or & & & 1,000 & 167 & & & & \\
\hline \multirow[t]{6}{*}{ LMB } & Harif, $^{73} 2008$ & 500 & 100 & 0.67 & 1,000 & & 20 & Yes & $75 \%$ & 119 \\
\hline & & & & & 1,000 & 167 & & & & \\
\hline & & 333 & 100 & 0.67 & & & 20 & & & \\
\hline & Davidson, ${ }^{71} 2006$ & 500 & 100 & 0.75 & 1,000 & & 20 & Yes & $81 \%$ & 63 \\
\hline & & & & & $\mathrm{I}, 000$ & 167 & & & & \\
\hline & & 333 & 100 & 0.67 & & & 20 & & & \\
\hline
\end{tabular}

Note: Study types include single agent cyclophosphamide studies, studies using COMP53 as the backbone of therapy, or those using variations the French LMB8954 studies with doxorubicin. Doses displayed are for the agents used in the first 4 months of the protocols. The last three studies alternated cycles of chemotherapy, using two or three different combinations of chemotherapy. Use of intrathecal medications (IT) is indicated. Survival percentage as shown is based the number of patients enrolled in the final column. Survival time varied studies with doxorubicin. Survival time was reported at one to five years of follow-up.

Abbreviations: COMP, cyclophosphamide, vincristine, methotrexate, and prednisone; LMB, lymphoma malignancy B-cell; meds, medications.

facilities, and establishing government sponsored support systems that include oncology and other chronic diseases. The authors are working to improve the infrastructure in their treatment centers in Kenya with the goal of safely and effectively delivering chemotherapy regimens shown to be most successful in high-income countries. However, since BL is after all a relatively rare disease, the formation of a global BL clinical trial network with standardized treatment protocols and level of care across hospitals would offer a platform upon which there could be a collective effort to advance the understanding of optimal therapeutic strategies. This would include efforts to empirically identify molecular pathways that lend themselves to rationally targeted chemotherapies with treatment trials that could prospectively determine if eBL survival is improved and relapse prevented.

\section{Factors that influence treatment outcomes: from socioeconomic factors to host genetics}

Beyond disease burden and selection of treatment protocols, there are multiple other factors that may influence treatment outcomes for eBL, ranging from socioeconomic factors to host genetics. These influences are important to consider, particularly in the absence of prognostic markers of response and survival validated for eBL in resource-limited settings. While prognostic markers of disease response and survival have been well established for non-Hodgkin's lymphoma in high-income settings (eg, tumor stage, serum LDH levels, presence or absence of central nervous system involvement), ${ }^{56}$ there are unfortunately more questions than answers when it comes to prognostic indicators for eBL.

It has been well established that the socioeconomic status of a child's family can have a profound impact on the choice to initiate treatment and/or adhere to prescribed care, particularly in low-income countries where abandonment of care is a common problem. ${ }^{57}$ The cost of chemotherapy alone is prohibitively expensive for most families in lowincome countries but the availability of national hospital insurance can improve patient retention. ${ }^{58}$ Additionally, because resource-constrained settings have relatively few treatment centers that are equipped to treat children with cancer, it is not uncommon for families to endure long (and expensive) commutes to receive therapy. These costs are often compounded during the child's in-hospital treatment as families experience losses in daily income while caregivers stay with their child, often for weeks or even 
months at a time. Together, these factors present significant barriers to care for families who are already struggling to make ends meet and the authors dream of a day when they could provide a subsidized place for families of hospitalized children to stay.

Host factors that are independent of tumor biology itself also play a role. Many children in resource-limited settings are malnourished even prior to a cancer diagnosis. The importance of nutritional status in children with cancer cannot be understated. Malnutrition has many effects on treatment, including decreased tolerance of chemotherapy, altered metabolism of antineoplastic drugs, increased infection rates, and poor clinical outcomes. ${ }^{59}$ Maintaining adequate nutrition throughout the course of treatment has been shown to have a positive impact on the risk of relapse and overall survival. ${ }^{60-63}$

Continuing to improve the survival of children with $\mathrm{BL}$ across Africa and other resource-limited settings will also require solutions that reach beyond the bedside. Studies offering social support for the child and family member caring for the child have shown significantly improved treatment adherence. This has included reimbursements for travel, lodging, and food, and, in some cases, partial or full subsidization of medical care, which further decreases the financial burden experienced by the family. If social support is available, a careful assessment of the family's resources should begin early, if not concomitantly with diagnostic evaluations. In Kenya, the authors have found that hiring a Case Manager (also referred to as a Patient Navigator) can alleviate some of the parents' stress by providing support while the child is in hospital. The Case Manager attends medical rounds with the doctors and is trained to re-explain the procedures and side effects of the medicine to the parents as well as bring questions from the parents back to the doctors. They can also assist with learning the hospital culture and how to manage the hospital system, including expectations for billing. The authors found that parents who worried about hospital expenses exceeding their means were more likely to abscond with their child before completing the course of treatment. In addition, the Case Manager contacts parents and reminds them of their child's outpatient clinic appointments after discharge and of the available transport reimbursement, which can improve follow-up care. While financial considerations are the major cause of treatment abandonment, insufficient communication between health providers and families can also contribute. In some cases, families stop treatment and abscond with early improvement in symptoms due to perceptions that the child is cured. In other cases, abandonment may be related to premature lost hope. Thus, efforts should be made throughout the child's hospitalization to explore guardians' perceptions of the patient's illness and offer counseling on their child's diagnosis and prognosis, as well as the duration and goals of treatment, particularly given the low awareness of pediatric cancer in the community and often limited knowledge of cancer as a potentially curable disease. Although there remains considerable focus on developing optimal chemotherapeutic regimens catered to individual patients, gains in survival will be limited until many of these additional factors known to influence adherence to care are addressed.

Finally, a cancer diagnosis in a child often leads to a variety of physical, social, and emotional challenges for the child, as well as their families. In addition to the financial issues previously discussed, families can face social stigma and isolation, shifting household responsibilities, and loss of employment, among other issues. Many groups have worked to address these issues by providing family and psychosocial support through a multidisciplinary approach involving pediatric hematologists/oncologists, pediatricians, pharmacists, and nurses, as well as local nongovernmental organizations and the community. Creating a community with parent and survivor support groups provides a platform for advocacy and broader engagement.

The role of host genetics in contributing to eBL risk, as well as treatment response, remains unclear at this time and deserves further investigation. An appropriately powered and controlled genetic epidemiologic study has yet to be done; however, with the affordability of next generation sequencing, molecular risk stratification, and the formation of a BL research and clinical trial network, such studies are on the horizon.

\section{Secondary complications}

Treatment-related toxicity remains a major barrier to improving survival among children with BL. The most common toxicity of chemotherapy regimens that are used to treat BL is myelosuppression, which results in an increased susceptibility to infection. While this is a risk of many chemotherapeutics in any setting, it is of particular concern in low-income settings where the ability to support patients through periods of neutropenia are limited. Compounding this issue is the fact that overcrowding is common in many hospitals in equatorial Africa because of the few locations where childhood cancer can be treated. ${ }^{4}$ Consequently, it is not unusual for children 
with neutropenia to be on the same ward (or worse, in the same bed) as a child with a communicable disease, making transmission of infections to the neutropenic patient even more likely. Chemotherapy-induced myelosuppression may also cause anemia and/or thrombocytopenia. While this toxicity can be easily supported in high-income settings through transfusion center services, the same is not true for most low-income settings where access to safe blood products may be neither reliable nor timely.

Chemotherapy-induced nausea and vomiting is another factor to consider when discerning an appropriate treatment protocol for a given setting. In resource-rich environments, malnutrition is a problem rarely encountered, and supportive antiemetics along with enteral or parenteral nutrition (if needed) are readily available. However, in low-income countries where many children are malnourished at baseline, the added challenge of chemotherapyinduced nausea and vomiting can make a difficult problem worse. Lack of reliable access to antiemetics may result in worsening malnourishment following chemotherapy administration. Furthermore, access to nasogastric tubes or enteral/parenteral formulas may be scarce or completely inaccessible.

Other chemotherapy-associated toxicities that are less common but deserve consideration when determining optimal treatment protocols include:

- mucositis (sometimes with lack of adequate analgesia), further complicating malnutrition

- skin necrosis from chemotherapy infiltration (when chemotherapy is peripherally administered)

- nephrotoxicity from cyclophosphamide and methotrexate

- vincristine-induced peripheral neuropathy

- cardiomyopathy from anthracyclines

- risk of secondary malignancy associated with use of alkylating agents and etoposide

- chemotherapy impact on pre-existing comorbidities (eg, HIV management).

\section{Conclusion: the way forward}

Over the past 10 years, BL treatment for African children has received renewed attention within the global community. During this period, the understanding of treatment regimens has evolved and appreciable progress has been made, from the single agent trials to the more intensive multidrug regimens that are more common today. Despite considerable investment in evaluating BL treatment options, clinical outcomes in many facilities in resource-limited settings remain suboptimal. Treatment-related mortality, late-stage of presentation, refusal of treatment, and abandonment of care remain major barriers to improving survival among children with eBL. Addressing these challenges is difficult, particularly in much of equatorial Africa where cancer care may not be as high priority as fighting acute infectious diseases and there continues to be a need for more basic, affordable health interventions. Formulating a BL treatment regimen should be made following a critical assessment of available resources and a strategic plan aimed at improving local conditions and increasing oncology specialized training in order to meet a basic standard of care upon which one can build. Careful attention should be paid to offering treatment with intensity matching the level of ancillary support available, including infrastructure and capacity to offer routine antibiotics, blood products, and nutritional support. However, optimal management of $\mathrm{BL}$ requires more than just delivering chemotherapy and supportive care; there is a need to develop and implement improved paradigms of care delivery, ones that incorporate multidisciplinary strategies to more effectively address social, economic, and system level factors influencing care. Survival outcomes will only improve with increased access to care, more timely and accurate diagnoses, referral and initiation of treatment, and provision of robust social support to ameliorate many of the socioeconomic factors that can lead to refusal or abandonment of care. Complementing these efforts will be the application of empirical evidence arising from projects, such as the Cancer Genome Atlas, ${ }^{64}$ that promise to elucidate biological pathways that can be targeted for the development of new and preferably less toxic chemotherapies used to cure BL.

\section{Disclosure}

The authors report no conflicts of interest in this work.

\section{References}

1. Burkitt D. A sarcoma involving the jaws in African children. Br J Surg. 1958;46(197):218-223.

2. Hesseling P, Israels T, Harif M, Chantada G, Molyneux E. Practical recommendations for the management of children with endemic Burkitt lymphoma (BL) in a resource limited setting. Pediatr Blood Cancer. 2013;60(3):357-362.

3. Parkin DM, Stiller CA, Draper GJ, Bieber CA. The international incidence of childhood cancer. Int J Cancer. 1988;42(4):511-520.

4. Magrath I, Steliarova-Foucher E, Epelman S, et al. Paediatric cancer in low-income and middle-income countries. Lancet Oncol. 2013;14(3): e104-e116.

5. Mwanda OW, Rochford R, Rainey J, Wilson ML. Challenges in the epidemiological and clinical aspects of Burkitt's lymphoma in Kenya: linking evidence and experience. East Afr Med J. 2004;8 Suppl: S111-S116. 
6. Haddow AJ. Age incidence in Burkitt's lymphoma syndrome. East Afr Med J. 1964;41:1-6.

7. Moormann AM, Snider CJ, Chelimo K. The company malaria keeps: how co-infection with Epstein-Barr virus leads to endemic Burkitt lymphoma. Curr Opin Infect Dis. 2011;24(5):435-441.

8. Epstein MA,Achong BG, BarrYM. Virus particles in cultured lymphoblasts from Burkitt's lymphoma. Lancet. 1964;1(7335):702-703.

9. Robertson ES, editor. Burkitt's Lymphoma. 1st ed. New York, NY: Springer; 2012.

10. Burkitt D, O'Conor GT. Malignant lymphoma in African children. I. A clinical syndrome. Cancer. 1961;14:258-269.

11. Burkitt D. A children's cancer with geographical limitations. Cancer Prog. 1963;92:102-113.

12. Haddow AJ. An improved map for the study of Burkitt's lymphoma syndrome in Africa. East Afr Med J. 1963;40:429-432.

13. Dalldorf G, Linsell CA, Barnhart FE, Martyn R. An epidemiologic approach to the lymphomas of African children and Burkitt's sarcoma of the jaws. Perspect Biol Med. 1964;7:435-449.

14. Burkitt D. A "tumour safari" in East and Central Africa. Br J Cancer. 1962;16:379-386.

15. Dalldorf G, Barnhart FE. Childhood leukemia, malaria and Burkitt's lymphoma. N Engl J Med. 1972;286(22):1216.

16. Rainey JJ, Mwanda WO, Wairiumu P, Moormann AM, Wilson ML, Rochford R. Spatial distribution of Burkitt's lymphoma in Kenya and association with malaria risk. Trop Med Int Health. 2007;12(8): 936-943.

17. Rainey JJ, OmenahD, Sumba PO, MoormannAM, RochfordR, Wilson ML. Spatial clustering of endemic Burkitt's lymphoma in high-risk regions of Kenya. Int J Cancer. 2007;120(1):121-127.

18. Moss DJ, Burrows SR, Castelino DJ, et al. A comparison of Epstein-Barr virus-specific T-cell immunity in malaria-endemic and -nonendemic regions of Papua New Guinea. Int J Cancer. 1983;31(6):727-732.

19. Whittle HC, Brown J, Marsh K, et al. T-cell control of Epstein-Barr virus-infected B-cells is lost during P. falciparum malaria. Nature. 1984;312(5993):449-450.

20. Donati D, Mok B, Chene A, et al. Increased B-cell survival and preferential activation of the memory compartment by a malaria polyclonal B-cell activator. J Immunol. 2006;177(5):3035-3044.

21. Piriou E, Asito AS, Sumba PO, et al. Early age at time of primary Epstein-Barr virus infection results in poorly controlled viral infection in infants from Western Kenya: clues to the etiology of endemic Burkitt lymphoma. J Infect Dis. 2012;205(6):906-913.

22. Greenwood B, Marsh K, Snow R. Why do some African children develop severe malaria? Parasitol Today. 1991;7(10):277-281.

23. Sumba PO, Kabiru EW, Namuyenga E, et al. Microgeographic variations in Burkitt's lymphoma incidence correlate with differences in malnutrition, malaria, and Epstein-Barr virus. Br J Cancer. 2010;103(11): 1736-1741.

24. van den Bosch CA. Is endemic Burkitt's lymphoma an alliance between three infections and a tumour promoter? Lancet Oncol. 2004;5(12): $738-746$.

25. Orem J, Maganda A, Mbidde EK, Weiderpass E. Clinical characteristics and outcome of children with Burkitt lymphoma in Uganda according to HIV infection. Pediatr Blood Cancer. 2009;52(4):455-458.

26. Schulz TF, Boshoff CH, Weiss RA. HIV infection and neoplasia. Lancet. 1996;348(9027):587-591

27. Davidson A, Wainwright RD, Stones DK, et al. Malignancies in South African children with HIV. J Pediatr Hematol Oncol. 2014;36(2): 111-117.

28. Stefan DC, Stones DK. Children with cancer and HIV infection: what is different about them? J Pediatr Hematol Oncol. 2013;35(8): 590-596.

29. Balfour HH Jr, Sifakis F, Sliman JA, Knight JA, Schmeling DO, Thomas W. Age-specific prevalence of Epstein-Barr virus infection among individuals aged 6-19 years in the United States and factors affecting its acquisition. J Infect Dis. 2013;208(8):1286-1293.
30. Condon LM, Cederberg LE, Rabinovitch MD, et al. Age-specific prevalence of Epstein-Barr virus infection among Minnesota children effects of race/ethnicity and family environment. Clin Infect Dis. Epub May 11, 2014.

31. Balfour HH Jr. Progress, prospects, and problems in Epstein-Barr virus vaccine development. Curr Opin Virol. 2014;6C:1-5.

32. Lindblade KA, Eisele TP, Gimnig JE, et al. Sustainability of reductions in malaria transmission and infant mortality in Western Kenya with use of insecticide-treated bednets: 4 to 6 years of follow-up. JAMA. 2004;291(21):2571-2580.

33. Mathenge EM, Gimnig JE, Kolczak M, Ombok M, Irungu LW, Hawley WA. Effect of permethrin-impregnated nets on exiting behavior, blood feeding success, and time of feeding of malaria mosquitoes (Diptera: Culicidae) in Western Kenya. J Med Entomol. 2001;38(4):531-536.

34. Strode C, Donegan S, Garner P, Enayati AA, Hemingway J. The impact of pyrethroid resistance on the efficacy of insecticide-treated bed nets against African anopheline mosquitoes: systematic review and metaanalysis. PLoS Med. 2014;11(3):e1001619.

35. Patel JD, Galsky MD, Chagpar AB, Pyle D, Loehrer PJ. Role of American Society of Clinical Oncology in low- and middle-income countries. J Clin Oncol. 2011;29(22):3097-3102.

36. Buckle GC, Collins JP, Sumba PO, et al. Factors influencing time to diagnosis and initiation of treatment of endemic Burkitt lymphoma among children in Uganda and Western Kenya: a cross-sectional survey. Infect Agent Cancer. 2013;8(1):36.

37. Razack R, Michelow P, Leiman G, et al. An interinstitutional review of the value of FNAB in pediatric oncology in resource-limited countries. Diagn Cytopathol. 2012;40(9):770-776.

38. Parham GP, Mwanahamuntu MH, Pfaendler KS, et al. eC3 - a modern telecommunications matrix for cervical cancer prevention in Zambia. J Low Genit Tract Dis. 2010;14(3):167-173.

39. Korir A, Mauti N, Moats P, et al. Developing clinical strength-ofevidence approach to define HIV-associated malignancies for cancer registration in Kenya. PLoS One. 2014;9(1):e85881.

40. Magrath I, Lee YJ, Anderson T, et al. Prognostic factors in Burkitt's lymphoma: importance of total tumor burden. Cancer. 1980;45(6): $1507-1515$

41. Garba IH, Ubom GA. Total serum lactate dehydrogenase activity in acute Plasmodium falciparum malaria infection. Singapore Med J. 2005;46(11):632-634.

42. Simon R. Clinical trial designs for evaluating the medical utility of prognostic and predictive biomarkers in oncology. Per Med. 2010;7(1): $33-47$.

43. Oettgen HF, Burkitt D, Burchenal JH. Malignant lymphoma involving the jaw in African children: treatment with methotrexate. Cancer. 1963;16:616-623.

44. Burkitt D, Hutt MS, Wright D. The African lymphoma: preliminary observations on response to therapy. Cancer. 1965;18:399-410.

45. Burkitt D. African lymphoma. Observations on response to vincristine sulphate therapy. Cancer. 1966;19(8):1131-1137.

46. Morrow RH, Pike MC, Kisuule A. Survival of Burkitt's lymphoma patients in Mulago Hospital, Uganda. Br Med J. 1967;4(5575):323-327.

47. Williams EH. Chemotherapy of Burkitt's lymphoma. Br Med J. 1969;2(5659):764.

48. Williams EH. A comparison of results achieved in treating two series of patients with Burkitt's lymphoma. Br J Cancer. 1971;25(1): $37-45$.

49. Ziegler JL. Chemotherapy of Burkitt's lymphoma. Cancer. 1972;30(6): $1534-1540$

50. Nkrumah FK, Perkins IV. Burkitt's lymphoma in Ghana: clinical features and response to chemotherapy. Int J Cancer. 1973;11(1):19-29.

51. Nkrumah FK, Perkins IV. Burkitt's lymphoma: a clinical study of 110 patients. Cancer. 1976;37(2):671-676.

52. Nkrumah FK, Perkins IV, Biggar RJ. Combination chemotherapy in abdominal Burkitt's lymphoma. Cancer. 1977;40(4):1410-1416. 
53. Anderson JR, Wilson JF, Jenkin DT, et al. Childhood non-Hodgkin's lymphoma. The results of a randomized therapeutic trial comparing a four-drug regimen (COMP) with a ten-drug regimen (LSA2-L2). N Engl J Med. 1983;308(10):559-565.

54. Patte C, Auperin A, Michon J, et al. The Societe Francaise d'Oncologie Pediatrique LMB89 protocol: highly effective multiagent chemotherapy tailored to the tumor burden and initial response in 561 unselected children with B-cell lymphomas and L3 leukemia Blood. 2001;97(11):3370-3379.

55. Hesseling PB, Broadhead R, Molyneux E, et al. Malawi pilot study of Burkitt lymphoma treatment. Med Pediatr Oncol. 2003;41(6): 532-540.

56. Sandlund JT, Downing JR, Crist WM. Non-Hodgkin's lymphoma in childhood. N Engl J Med. 1996;334(19):1238-1248.

57. Njuguna F, Mostert S, Slot A, et al. Abandonment of childhood cancer treatment in Western Kenya. Arch Dis Child. Epub March 28, 2014.

58. Mostert S, Njuguna F, van de Ven PM, et al. Influence of healthinsurance access and hospital retention policies on childhood cancer treatment in Kenya. Pediatr Blood Cancer. 2014;61(5):913-918.

59. Lu TX, Mai WY, Teh BS, et al. Initial experience using intensitymodulated radiotherapy for recurrent nasopharyngeal carcinoma. Int J Radiat Oncol Biol Phys. 2004;58(3):682-687.

60. Donaldson SS, Wesley MN, DeWys WD, Suskind RM, Jaffe N, van Eys J. A study of the nutritional status of pediatric cancer patients. Am J Dis Child. 1981;135(12):1107-1112.

61. Lobato-Mendizabal E, Ruiz-Arguelles GJ, Marin-Lopez A. Leukaemia and nutrition. I: malnutrition is an adverse prognostic factor in the outcome of treatment of patients with standard-risk acute lymphoblastic leukaemia. Leuk Res. 1989;13(10):899-906.

62. Mejia-Arangure JM, Fajardo-Gutierrez A, Reyes-Ruiz NI, et al. Malnutrition in childhood lymphoblastic leukemia: a predictor of early mortality during the induction-to-remission phase of the treatment. Arch Med Res. 1999;30(2):150-153.

63. Viana MB, Murao M, Ramos G, et al. Malnutrition as a prognostic factor in lymphoblastic leukaemia: a multivariate analysis. Arch Dis Child. 1994;71(4):304-310.
64. Cancer Genome Atlas Network. Comprehensive molecular characterization of human colon and rectal cancer. Nature. 2012; 487(7407):330-337.

65. Kazembe P, Hesseling PB, Griffin BE, Lampert I, Wessels G. Long term survival of children with Burkitt lymphoma in Malawi after cyclophosphamide monotherapy. Med Pediatr Oncol. 2003;40:23-25.

66. Traore F, Coze C, Atteby JJ, et al. Cyclophosphamide monotherapy in children with Burkitt lymphoma: a study from the FrenchAfrican Pediatric Oncology Group (GFAOP). Pediatr Blood Cancer. 2011;56:70-76.

67. Hesseling P, Molyneux E, Kamiza S, Israels T, Broadhead R. Endemic Burkitt lymphoma: a 28-day treatment schedule with cyclophosphamide and intrathecal methotrexate. Ann Trop Paediatr. 2009;29:29-34.

68. Koffi GK, Tolo A, Nanho DC, et al. Results of treatment with CMA, a low intermediate regimen, in endemic Burkitt lymphomas in subSaharian Africa: experience of Cote d'Ivoire. International journal of hematology. 2010;91:838-843.

69. Ngoma T, Adde M, Durosinmi M, et al. Treatment of Burkitt lymphoma in equatorial Africa using a simple three-drug combination followed by a salvage regimen for patients with persistent or recurrent disease. Br J Haematol. 2012;158:749-762.

70. Hesseling P, Broadhead R, Mansvelt E, et al. The 2000 Burkitt lymphoma trial in Malawi. Pediatr Blood Cancer. 2005;44:245-250.

71. Davidson A, Desai F, Hendricks M, et al. The evolving management of Burkitt's lymphoma at Red Cross Children's Hospital. S Afr Med J. 2006;96:950-954.

72. Hesseling P, McCormick P, Kouya F, et al. Burkitt lymphoma: residual abdominal tumor volume after induction therapy correlates with outcome. Pediatr Blood Cancer. 2010;55:761-762.

73. Harif M, Barsaoui S, Benchekroun S, et al. Treatment of B-cell lymphoma with LMB modified protocols in Africa-report of the French-African Pediatric Oncology Group (GFAOP). Pediatr Blood Cancer. 2008;50:1138-1142.
Blood and Lymphatic Cancer: Targets and Therapy

\section{Publish your work in this journal}

Blood and Lymphatic Cancer: Targets and Therapy is an international, peer-reviewed, open access journal focusing on blood and lymphatic cancer research, identification of therapeutic targets and the optimal use of preventative and integrated treatment interventions to achieve improved outcomes, enhanced survival and quality of life for the

\section{Dovepress}

cancer patient. The manuscript management system is completely online and includes a very quick and fair peer-review system. Visit http://www.dovepress.com/testimonials.php to read real quotes from published authors. 